Instructions for authors, subscriptions and further details:

http://brac.hipatiapress.com

\title{
El Relato de un Proceso: Signs that Say what You Want them to Say and not Signs that Say what Someone Else Wants You to Say (1992-93) de Gillian Wearing
}

Montserrat López Páez ${ }^{1}$

1) Universitat de Barcelona. España

Date of publication: February $3^{\text {rd }}, 2016$

Edition period: February 2016 - June 2016

To cite this article: López Páez, M. (2015). El Relato de un Proceso: Signs that Say what You Want them to Say and not Signs that Say what Someone Else Wants You to Say (1992-93) de Gillian Wearing. Barcelona, Research, Art, Creation, 4(1), 32-49. doi: 10.17583/brac.2016.1409

To link this article: http://dx.doi.org/10.17583/brac.2016.1409

\section{PLEASE SCROLL DOWN FOR ARTICLE}

The terms and conditions of use, except where otherwise noted, are related to the Open Journal System and to Creative Commons Attribution License (CCBY). The indication must be expressly stated when necessary. 
BRAC - Barcelona Research Art Creation. Vol. 4 No. 1, February 2016, pp. 32-49

\section{The Story of a Process: Signs that Say what You Want them to Say and not Signs that Say what Someone Else Wants You to Say (1992-93), By G. Wearing}

Montserrat López Páez

University of Barcelona

(Received: 6 February 2015; Accepted: 11 December 2015; Published: 3 February 2016)

\section{Abstract}

This article is an approximation to the project Signs that say what you want them to say and not Signs that say what someone else wants you to say (1992-93), by G. Wearing, from the statement of the process or the history of the project. Conversations with the artist, published between 1997 and 1999, allow us the reconstruction of the process in order to apprehend, from a more wide perspective, a work of reference to the artistic current practice.

Keywords: Gillian Wearing, artistic process, artistic project, photographic portrait, documentary fiction, relational art, contemporary art

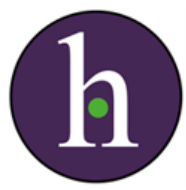


BRAC - Barcelona Research Art Creation. Vol. 4 No. 1, February 2016, pp. 32-49

\section{El Relato de un Proceso: Signs that Say what You Want them to Say and not Signs that Say what Someone Else Wants You to Say (1992-93) de Gillian Wearing}

Montserrat López Páez

Universidad de Barcelona

(Recibido: 6 Febrero 2015; Aceptado: 11 Diciembre 2015; Publicado: 3 Febrero 2016)

\section{Resumen}

El presente artículo es una aproximación al proyecto Signs that say what you want them to say and not Signs that say what someone else wants you to say (1992-93), de G. Wearing, a partir del relato del proceso o la historia del proyecto. Conversaciones con la artista, publicadas entre 1997 y 1999, nos permiten la reconstrucción del proceso a fin de aprehender, desde una perspectiva más amplia, una obra de referencia para la práctica artística actual.

Palabras clave: Gillian Wearing, proceso artístico, proyecto artístico, retrato fotográfico, ficción documental, arte relacional, arte contemporáneo.

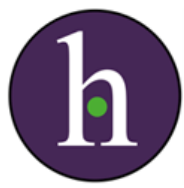




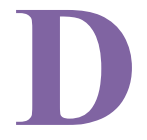

urante 1992 y 1993, la artista Gillian Wearing (Birmingam, 1963) emprende largos paseos por el centro de Londres y, mientras tanto, se detiene en enclaves elegidos al azar, aborda a los viandantes y les propone escribir en una hoja de papel en blanco cualquier asunto o idea que se les ocurra para, acto seguido, fotografiarlos junto a sus palabras en mano. Se trata del proyecto Signs that say what you want them to say and not Signs that say what someone else wants you to say (Signos que dicen lo que quieres decir y no signos que dicen lo que otra persona quiere que digas).

Este artículo es una aproximación a este proyecto de Wearing, que en adelante se mencionará como Signs..., a partir de la reconstrucción de su proceso de creación (antecedentes, primeras tomas, experiencia compartida...). La artista concibe dicho proceso como parte integrante de la obra, al cual acompaña y conforma a cada paso, aunque éste, la mayoría de las veces, no pueda mostrarse al público. De hecho, la propia Wearing reivindica constantemente de Signs... esta característica en las conversaciones en las que refiere la obra, la mayoría de ellas publicadas entre 1997 y 1999. Estas conversaciones son la fuente principal y guía del siguiente relato, en un intento por trasladar el formato conversacional de Wearing en Signs... al presente texto.

Empecemos, pues, el relato por los antecedentes. Éste es el primer gran proyecto de una jovencísima Wearing, quien justo en 1990 finalizó sus estudios de arte en la Goldsmiths' College de la Universidad de Londres. Durante esta etapa como en sus anteriores estudios, Wearing se había interesado indistintamente por la pintura, la instalación y el vídeo, sin lograr resultados satisfactorios. A mi entender, no se trataba sólo del cómo o de encontrar la forma de decir, sino que más bien se trataba de qué decir o de hallar el contenido mismo de su obra.

Precisamente el qué, o el contenido de sus proyectos, guarda una estrecha relación con un particular planteamiento de Wearing respecto a las personas y a las relaciones que se establecen entre ellas. Desde muy joven, la artista se sintió atraída por la gente, y en particular por los vínculos que establecían entre sí, interés siempre mediatizado por su propia experiencia personal. Ese será el objeto que iba a revelarse en el proyecto Sings..., un tema que emergerá como el leit motiv de la producción de la artista: la comunicación interpersonal, o si se prefiere, la relación con el otro. 


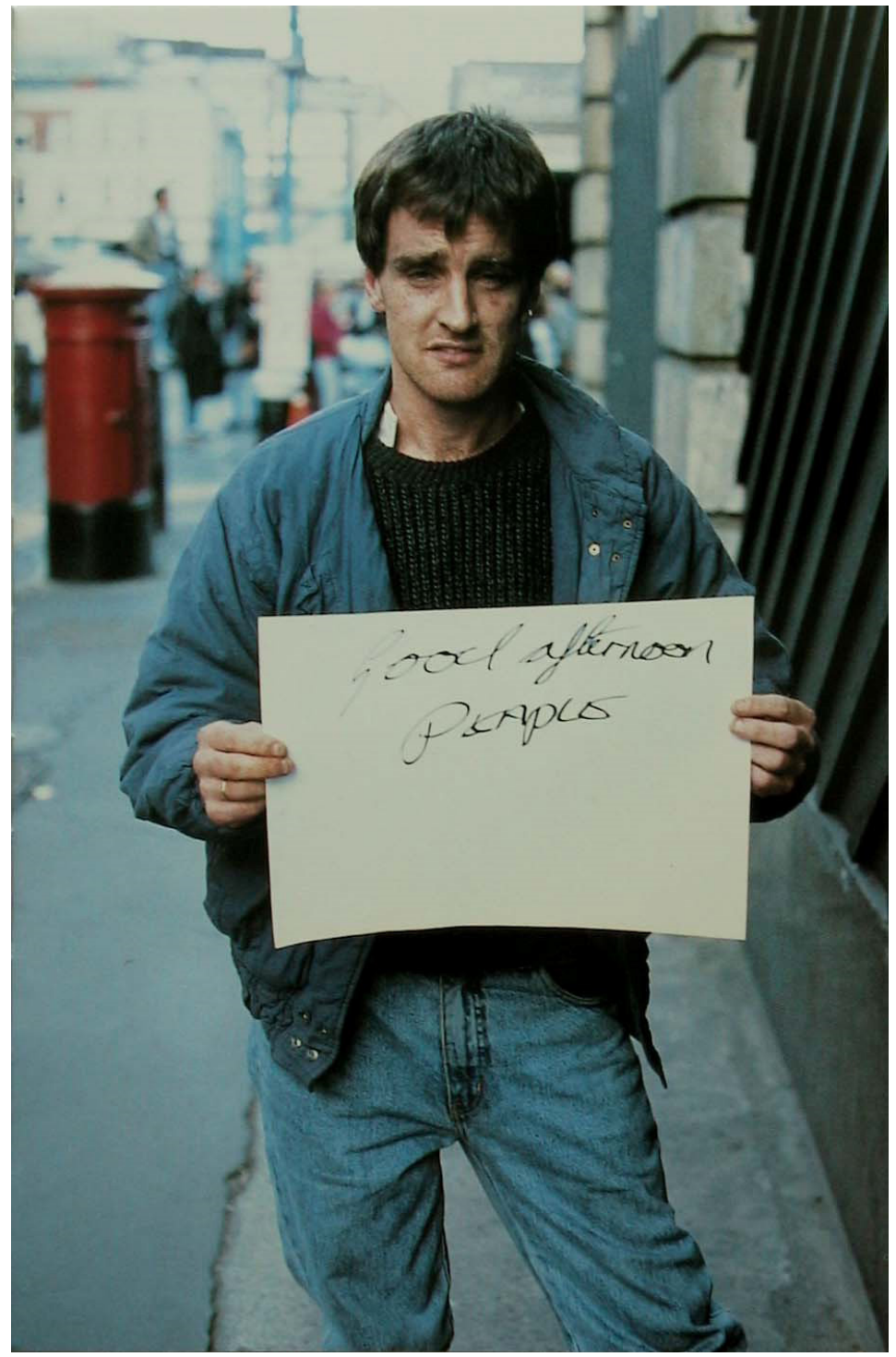

Figura 1. Gillian Wearing, Signs that say what you want them to say and not signs that say what someone else wants you to say, 1992-93.

Fuente: (Wearing, 1997) Signs that say what you want them to say and not signs that say what someone else wants you to say, Maureen Paley/ Interim Art, London. 
Antes de comenzar Sings..., Wearing, incluso deseando enormemente acercarse a la gente, se reconocía abiertamente tímida. Ella misma atribuye a su introversión el inicial retraso en la ejecución del proyecto. Su mínima capacidad de relacionarse era, al parecer, justo la opuesta que precisaba Sings... para llevarse a cabo. En 1999, en una conversación sostenida con Donna De Salvo, la artista se refiere a este tema y nos relata un intento fallido previo a Sings..., unas pruebas realizadas en su estudio con frases que ella misma escribió y que hizo sostener a sus amigos para así fotografiarlos. Pero aquello "no funcionó" (Wearing \& Ferguson,1999, p. 8), en palabras de la propia artista.

I was quite shy when I first started that project and I couldn't approach anyone -or thought I couldn't anyway. I originally started off by writing things myself, handing them to people I knew and asking them to hold them up. I set it up indoors and there was no real aesthetic or structure to it. It wasn't working ${ }^{1}$.

Me permito romper el hilo de la narración sobre el curso del proyecto Sings..., porque vale la pena señalar aquí que el primero en destacar el parecido visual entre este proyecto de Wearing y la Variable Piece $N^{\circ} 70$ (1971), de Douglas Huebler (1924-1997), fue Russell Ferguson (Wearing \& Ferguson, 1999, p. 48). De hecho, las tres últimas frases del párrafo anterior de Wearing podrían asimismo explicar la Variable Piece $N^{\circ} 70$ de Huebler y, ciertamente, la similitud entre ambos trabajos va mucho más allá de un "cierto parentesco visual", tal y como sostiene J. V. Aliaga en su artículo Gente normal. Sobre la obra de Wearing (Wearing, 2011, p. 11).

Huebler, en su Variable Piece $N^{\circ} 70$, invita a personas a su estudio para hacerlas posar ante la cámara sujetando en sus manos alguna de sus "declaraciones dactilográficas", tales como "una persona que está aburrida de su vida pero que jamás lo admitiría' o 'una persona que sea tan bonita como un cuadro'. Lo que a menudo se obvia -no en el caso de R. Ferguson, aunque éste se limita a apuntarlo en un pie de foto adjunto a su artículo- es que esas fotografías forman parte de un proyecto más amplio, que Huebler se propuso continuar hasta el fin de sus días, consciente de lo irrealizable de su propuesta, un "proyecto de inventario fotográfico de la especie humana" (Huebler, 1992, p. 119).

Éste es, en mi opinión, el punto de máxima confluencia entre Huebler y 
Wearing, aunque con diferentes matices, que se escapan del objetivo de este estudio: un interés desmedido por la especie humana o por el hombre, pero no por el hombre en mayúsculas sino por el hombre y la mujer en minúsculas. Ambos comparten una apasionamiento irrefrenable por las personas, por la gente normal y corriente, por el otro.

En el caso de Wearing, su interés por el otro data de la infancia. En varias entrevistas, la artista alude a esa fascinación natural por las personas. Quizás sea durante una conversación mantenida con Ben Judd en 1997 donde mejor explicita esa pasión personal (Judd, 1997, p. 4-5):

I always have been interested in people, even when I was a young kid. So it seems for me quite a natural thing that I'm interested in. I'd like to find out as many facets as possible about people. I'm interested in people more than I am in myself, maybe that's what it is ${ }^{5}$.

Inmediatamente después de finalizar sus estudios universitarios, Wearing comienza a trabajar a media jornada en un centro de arte, empleo que le proporciona un mínimo de ingresos. Tras varias tentativas amateur en vídeo y movida por esa particular atracción suya por las personas, Wearing se arma de valor y logra vencer su timidez. Al otro lado de la calle donde trabaja se encuentra el Regent's Park. Allí consigue el primer registro (Wearing \& Ferguson, 1999, p. 8) para el proyecto que más tarde denominará Signs...

Then one day I happened to go into Regent's Park, and I realized that I just had to do it: I had to pluck up my courage and approach strangers. The firts person who came along was this older woman. I asked her to write something down and she wrote, "I really love Regent's Park". From that moment I just knew it would work.

En una entrevista con Leo Edelstein fechada en 1998, Wearing explica más detalles sobre aquella primera experiencia y registro número uno (Edelstein \& Wearing, 1998):

I remember when she wrote it down the way she was looking -she was in her sixties and she had a lovely face- and I thought it was great, and quite banal. She held it up and was winter and I knew without even pressing the button that it worked, and I thought that I'd start asking more people. 
El proyecto Signs... estaba en marcha y tras aquel primer registro en el parque, se suceden hasta un total aproximado de 600 registros más, siempre siguiendo el mismo proceder. Wearing salía a la calle, se detenía en cualquier esquina e interpelaba a una persona cualquiera con la siguiente consigna: "Soy una artista y estoy llevando a cabo un proyecto en el que solicito a la gente que me escriba algo en una hoja de papel en blanco" (Wearing \& Ferguson, 1999, p. 9).

I'd stand on a street corner, anywhere. I'd probably be there for about ten or fifteen minutes. I didn't want to stay there for too long, as people would begin to see me as part of the scenery or as that odd girl, and I didn't want them to come back. I stopped people and just said, 'I'm an artist, and I'm doing a project where I ask people to write something on a piece of paper $^{8}$.

Tres años después de aquel 'I Really Love Regent's Park' y durante el año siguiente de haber cesado en sus capturas, Wearing sigue considerando todo aquel material compilado como documentación, sin saber muy bien qué hacer con ella. De hecho, la ausencia de intenciones a priori es una constante que se mantiene a lo largo de todo el proceso de trabajo, sin que ello influya de manera negativa en el desarrollo del mismo. Y aunque ésta no sea la manera habitual de trabajar de la artista, ella misma reconoce que en aquella ocasión así ocurrió. 




Figura 2. Gillian Wearing, Signs..., 1992-93.

Fuente: (Wearing, 1997) Signs ..., Maureen Paley/ Interim Art, London. 
Sólo desde una cierta distancia para con el proyecto, consigue Wearing extraer los significados de su Signs..., aún y habiendo producido una segunda versión, fechada en 1994, en Nápoles. En 1997 se celebra la primera exposición de una parte considerable del proyecto en la Galería Maureen Paley/Interim Art de Londres y, con motivo de esta, se edita una publicación de idéntico nombre (Wearing, 1997), que incluye una selección de 46 imágenes, precedidas por una breve introducción de la artista. En ella la artista nos proporciona una clave de lectura fundamental de la obra.

When most people are stopped in the street they expect to be asked questions usually concerned with either a product, money, a survey, a personality test or directions.

To be asked only to write something, anything, presents a challenge and creates a totally different relationship to the person posing the question. The bizarre request to be 'captured' on film by a complete stranger is compounded by a non-specific space; the blank piece of paper, which almost an unexposed film.

Perhaps the fascination in the relations between the person and their slogan in the confidence or diffidence of the people being 'imaged' in the first place.

This image interrupts the logic of photo-documentary and snapshot photography by the subjects clear collusion and engineering of their own representation.

Antes de intentar dar un significado a Signs..., solicitaría al lector prestar atención a cada uno de los signos que enuncian las personas interceptadas por Wearing. Seguidamente se adjunta una selección de los registros compilados en la publicación antes citada. 


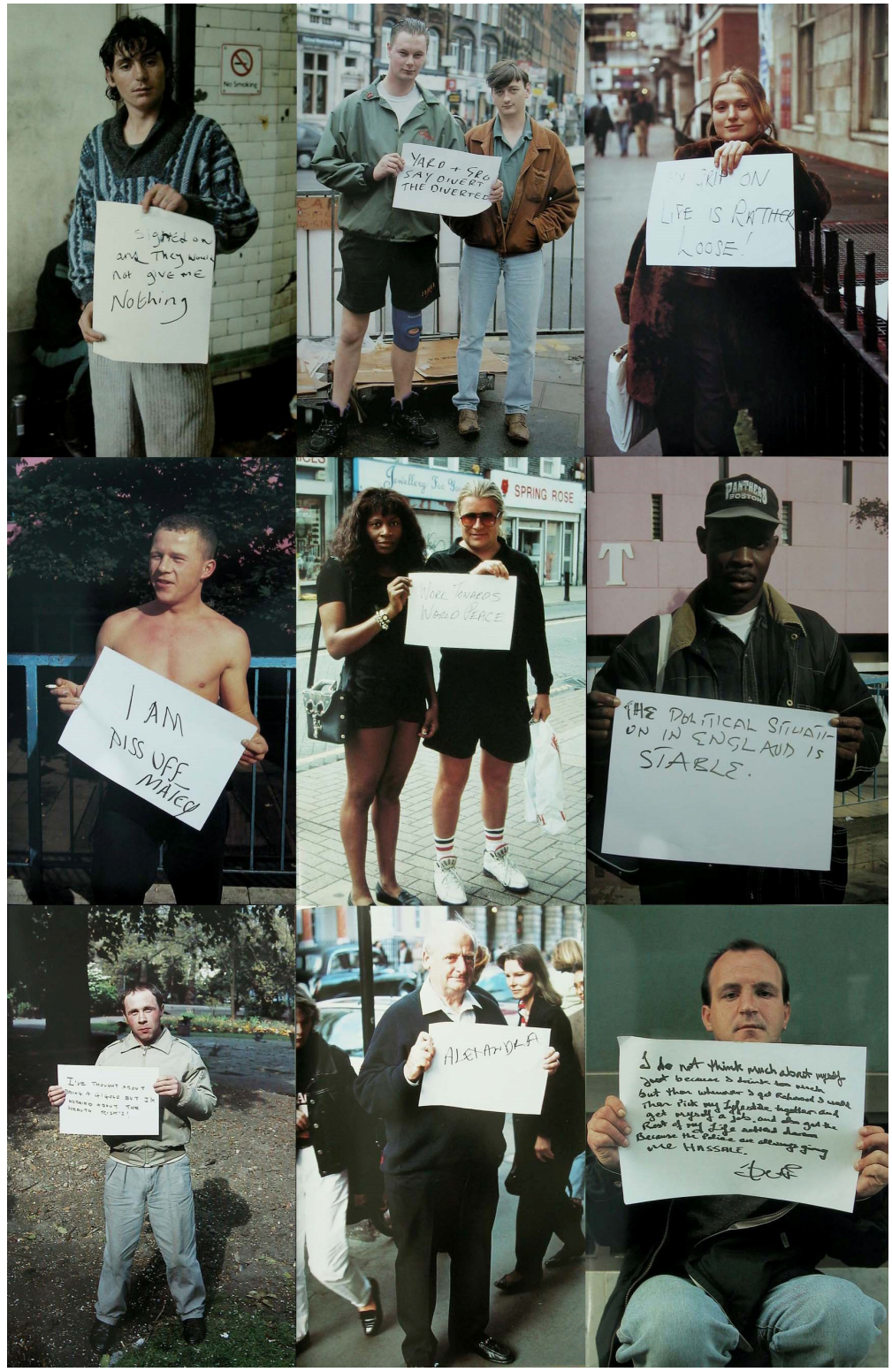

Figura 3. Gillian Wearing, Signs..., 1992-93.

Fuente: (Wearing, 1997) Signs ..., Maureen Paley/ Interim Art, London. 


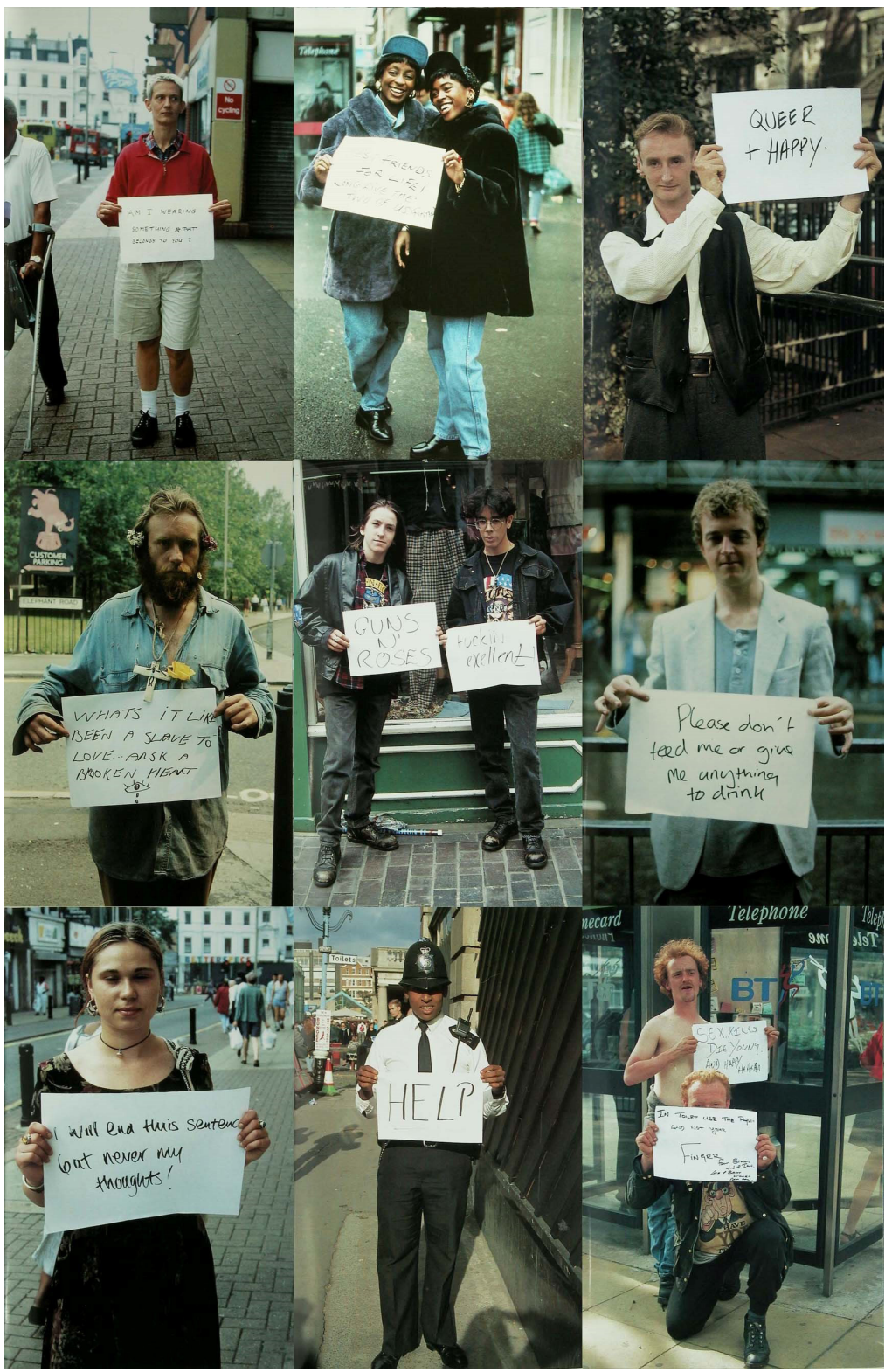

Figura 4. Gillian Wearing, Signs..., 1992-93.

Fuente: (Wearing, 1997) Signs ..., Maureen Paley/ Interim Art, London. 
Ciertamente las personas nos dicen cosas asombrosas. Así lo apunta Wearing al ser interrogada por De Salvo (Wearing \& Ferguson, 1999, p. 11) al respecto:

It was the sign that said, 'I've thought about being a gigolo but I'm worried about the health risks'. When I looked at these photographs of what people had said, I kept thinking. 'God, you said that!' For weeks and weeks there were these people saying the most amazing things.

Los dichos de Signs... son en sí mismos sorprendentes, y ello confiere excepcionalidad a todos y cada uno de los registros por sí solos. ¿Y por qué? En primer lugar, resultan inesperados porque Wearing parte de la realidad y, como máxima, la realidad siempre supera a la ficción. En segundo lugar, lo verdaderamente extraordinario, la ruptura de la convención, surge desde el mismo punto de arranque del proyecto. Me refiero a la sencilla y original, a un tiempo, propuesta que Wearing lanza a la gente o al público, observador y actor, en este caso, del devenir del proyecto. Como explica Wearing en su texto, cuando hoy en día alguien te para por la calle, te insta a dar una opinión acerca de un producto, a realizar una encuesta o a una limosna, sin más. Siempre la demanda persigue un fin mercantil. Wearing, en cambio y a diferencia de lo habitual, aborda a los viandantes y les propone lo insospechable, lo inimaginable: -Cuéntame cualquier cosa acerca de ti, lo primero que se te ocurra. La petición de Wearing consiste en todo lo contrario. Ella sólo pide: -Dame de ti.

Desde la imprevista petición inicial, así como a lo largo de todo el proceso, la imaginación se halla presente y juega un papel decisivo en Signs... La inventiva precede a cualquier empresa artística, o al menos así debiera ser, y Wearing parece intuirlo. La imaginación actúa también en este proyecto como el detonante de un tipo de relación muy especial que se propicia entre la artista y la personas interceptadas durante el devenir del proyecto. Y como resultado de ello, la imaginación es igualmente la responsable de que se genere la sorprendente serie de respuestas. Wearing está convencida de que la creatividad no es patrimonio exclusivo de los artistas, o si lo es, entonces considera al otro también artista.

Wearing calcula que un $85 \%$ de la gente que detuvo por la calle accedió a su inusual petición. Y no sólo accedieron sin dilación, sino que además se mostraron honestos ante la cámara de un extraño que les hacía una no menos extravagante proposición. $\mathrm{Y}$, aunque la artista prefería no permanecer 
durante mucho tiempo en un mismo lugar de la calle, para que los futuros participantes no supieran de sus predecesores, también nos explica como las conversaciones, en ocasiones, se prolongaban, llegando incluso con alguno de sus retratados a compartir sus más íntimos secretos.

Nótese que en el párrafo anterior introduje por primera vez el término "retratados", antes incluso de emplear el de "retrato". Debía ser en este orden, y no otro, para respetar el proceso de aprendizaje personal de la artista, que coexiste con el proceso de creación del proyecto. Para Wearing, antes que nada, se halla la fascinación por las personas. "Lo más importante es la relación que se establece entre nosotros" o "lo más importante es la experiencia compartida", son frases a menudo enunciadas por Wearing en sus conversaciones. Después vino el cómo hacer funcionar una cámara, los asuntos técnicos. Y, finalmente, aparece el género artístico implícito en sus registros: el retrato. "Estoy interesada por el retrato".

Así pues, el retrato de Wearing, como se apuntó al comienzo de este artículo, prioriza la relación con el otro y se propone principalmente otorgarle voz al otro. Éste es un propósito del quehacer de Wearing: dar voz a quienes no disponen de ella (Wearing, 2011, p. 26).

Cuando empecé a fotografiar a la gente, pensé que lo que tenía que decir era muy poco y que tenía mucho que aprender de escuchar y observar a los demás. A través de la televisión y de los anuncios nos bombardean con determinados mensajes, y pensé que había muchas cosas que se quedaban en el tintero y que había algunas personas a las que nunca llegabas a oír. 


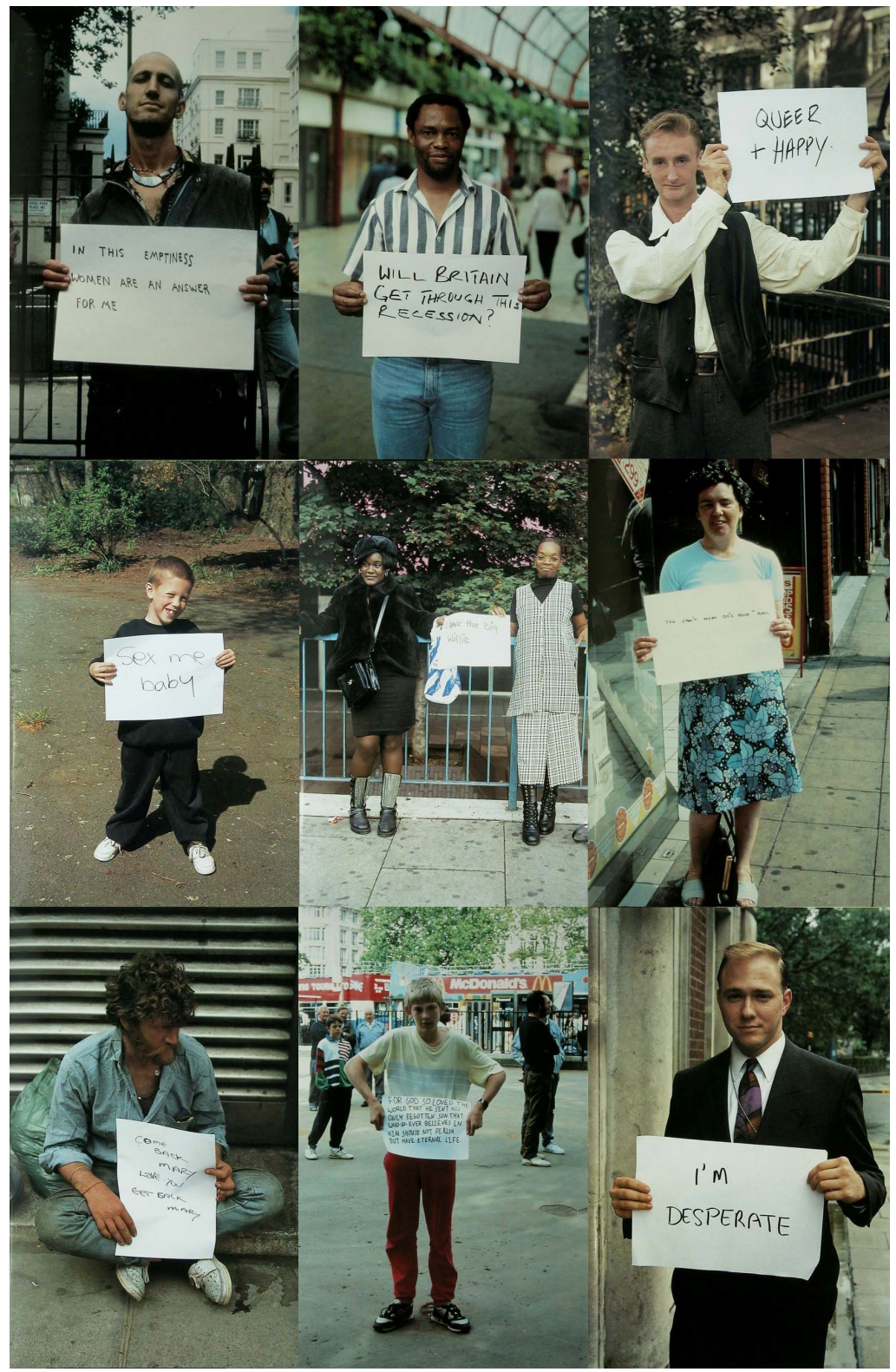

Figura 5. Gillian Wearing, Signs..., 1992-93.

Fuente: (Wearing, 1997) Signs..., Maureen Paley/ Interim Art, London. 
Un retrato, con un telón de fondo: Londres, 1990. Wearing habla de su vínculo con esta ciudad en la conversación con Leo Edelstein (Edelstein \& Wearing, 1998).

... when I came to London from Birmingham in 1983 it seemed so cosmopolitan and it's always been fascinating. When I was younger I was always thinking about how much I'd like to be in London. I've always been completely in awe of the place. I've had a bit of a love affair with it. Even though I'm used to living here now, I can still go out and think, what a great range of activity and people.

Pero la elección del centro de Londres como área de intervención del proyecto Signs... concede al mismo una carga significativa doble. Para el antropólogo Marc Augé, en el centro de una ciudad europea confluyen el "lugar antropológico tradicional" y el "espacio contemporáneo urbano" (Augé, 1998). Para este autor, ambos, el lugar antropológico y el espacio contemporáneo urbano, aunque en distinta medida, tienen un rasgo de itinerarios y recorridos personales, de lugares de relaciones y de intercambios (económicos principalmente, y de monumentos históricos, bien sean religiosos o políticos). La localización, pues, de Signs..., aunque elegida por Wearing sin pretensiones, es una localización física y social de base, inherente a un paisaje humano en movimiento constante, y en consecuencia, el marco ideal para un retrato humano, urbano y contemporáneo.

Precisamente, por estos tres calificativos, tanto el público-actor del proyecto se presta a participar en el mismo, como el público-observador de la exposición del mismo se siente idénticamente atraído por él. Cuando la obra es contemplada por el público-observador, éste puede muy fácilmente empatizar con el otro e incluso ponerse en su lugar, porque no difiere tanto de éste: humano, urbano, contemporáneo...

Un retrato directo, sincero, capturado por una mirada que se pretende libre de prejuicios y que se nos traslada como tal. Wearing intenta desembarazar sus registros de seísmos, clasismos, maniqueísmos... y hace aparecer ante la cámara a todos iguales y distintos entre sí, poniendo énfasis en la imaginación de la mirada que recorre los registros y que ella reclama como esa parte izquierda presente en todos sus referentes. Un retrato, pues, en el que los retratados parece que nos hablan a través del cuadro. Por esta particularidad varios autores han visto en Diana Arbus una precursora del trabajo de Wearing, aunque ésta última explica que precisamente ha sido esta atribución la que le 
llevó a interesarse y estudiar la producción de la primera, de quien no había tenido noticias hasta después de llevar a cabo su trabajo Signs...

Un retrato llamado -recordemos el nombre tras el abuso de su abreviación a lo largo de este texto-Signos que dicen lo que quieres decir y no signos que dicen lo que otra persona quiere que digas, y en cuyo título hallamos la mejor clave de lectura que la artista puede facilitarnos: signos y no signos, lo que quieres decir y lo que otra persona quiere que digas. La denominación Signs... nos habla de la existencia y de la inexistencia de los signos, de presencia y ausencia y, de igual modo, nos habla de esencias y de apariencias, y de nuevo de presencia y ausencia.

Se trata de un retrato dual, desde su nombre hasta la configuración de todos y cada uno de los registros, unidades significativas compuestas de un signo lingüístico y de un signo icónico, en contraposición permanente entre sí. Lo que apunta el texto de cada registro parece desmentirlo la imagen del mismo, y viceversa. La dualidad de signos apela a la dicotomía consubstancial al ser humano, a saber: ser/ parecer, no ser/no parecer, lo que somos y lo que deseamos ser, lo que no somos y lo que no deseamos ser, en definitiva, presencia y esencia.

Un retrato, para acabar, que apela directamente a una de las condiciones por excelencia de la especie humana: la contradicción. Cuando la reconocemos en los otros, nos reconocemos a nosotros mismos. A mi juicio, esto es lo que en realidad nos dice Signs...: miremos al otro, o miremos al otro para comprendernos un poco mejor a nosotros mismos. 


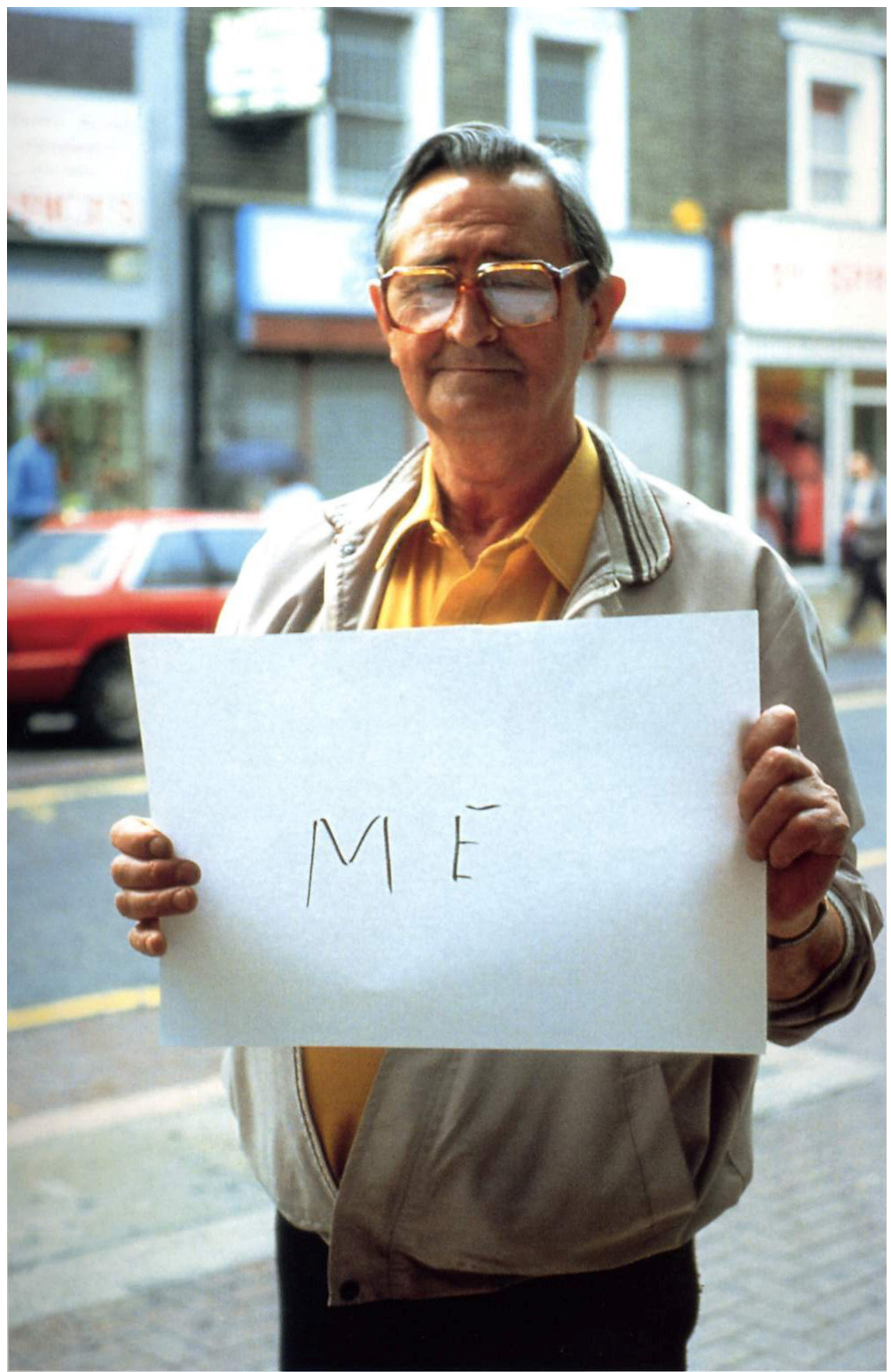

Figura 5. Gillian Wearing, Signs..., 1992-93.

Fuente: (Wearing, 1997) Signs ..., Maureen Paley/ Interim Art, London. 


\section{Referencias}

Augé, M. (1998) Los "no lugares". Espacios del anonimato. Barcelona: Gedisa.

Edelstein, L.; Wearing, G. (1998) Gillian Wearing en conversación con Leo Edelstein (1998) Journal of Contemporary Art. [Consult. 2015/02/05]

Disponible en <URL: http://www.jca-online.com/wearing.html

Huebler, D. (1992) Douglas Huebler. Variable, etc, Fons Regional D’Art

Contemporain/ Limosin Publications, Limoges.

Judd, B. (1997) Interview with Gillian Wearing, Untitled, $\mathrm{n}^{\circ}$ 12: invierno 1996-1997, London.

Wearing, G. (2011) Gillian Wearing. Madrid: Fundación la Caixa/ Centro Galego De Arte Contemporáneo.

Wearing, G.; Ferguson, R. (1999) Gillian Wearing. London: Phaidon.

Wearing, G. (1997) Signs that say what you want them to say and not signs that say what someone else wants you to say. London: Maureen Paley/ Interim Art.

Wearing, G.; Button, V (1997) Turner Prize 1997. London: Tate.

Montserrat López Páez: Artista visual. Professora i Doctora en Belles Arts per la Universitat de Barcelona (UB).

Contact Address: Universitat de Barcelona. Facultat de Belles Arts, Departament de Dibuix. Pau Gargallo, 4. 08028 Barcelona (Espanya).

E-mail address: montselopez@espaiefimer.com 